\title{
Influence of low-temperature annealing on the state of CdTe surface
}

\author{
O.A. Parfenyuk ${ }^{1}$, M.I. Ilashchuk ${ }^{1}$, S.M. Chupyra ${ }^{1}$, V.R. Burachek ${ }^{1}$, \\ D.V. Korbutyak ${ }^{2}$, S.G. Krylyuk ${ }^{2}$, N.D. Vakhnyak ${ }^{2}$ \\ ${ }^{1}$ Yu. Fed'kovych Chernivtsi National University, 2, Kotsyubinsky str., 58012 Chernivtsi, Ukraine \\ ${ }^{2} V$. Lashkaryov Institute of Semiconductors Physics, NAS of Ukraine, \\ 41, prospect Nauky, 03028, Kyiv, Ukraine \\ Phone/fax: +(380 44) 5256391, e-mail:div47@isp.kiev.ua
}

\begin{abstract}
Influence of low-temperature annealing on electrical and luminescent properties of semi-insulating $n$-CdTe:Sn is studied. It is shown that annealing at $T \geq 453 \mathrm{~K}$ modifies a near-surface layer of the crystals and this enhances the hole component of the conductivity. It is explained by $\mathrm{Cd}$ evaporation and subsequent enrichment of the near-surface layer with cadmium vacancies. Results of electrical measurements are confirmed by our low-temperature photoluminescence experiments.
\end{abstract}

Keywords: cadmium telluride, annealing, photoluminescence, surface, vacancy.

Manuscript received 01.06.05; accepted for publication 25.10.05.

\section{Introduction}

Nowadays, cadmium telluride (CdTe) is one of the most investigated $\mathrm{A}^{\mathrm{II}} \mathrm{B}^{\mathrm{VI}}$ semiconductor materials. Specific physical characteristics of this compound make it very attractive for various applications, in particular as detectors of hard radiation [1]. Information on the state of CdTe surface and its change after different treatments is very important for both fundamental and applied developments, including contact deposition and production of devices.

As far as the modification of CdTe surface and a near-surface layer due to influence of different external factors is concerned, two main directions can be distinguished. The first one is investigations of the oxidation mechanisms of $\mathrm{CdTe}$ and formation of rectifying metal-oxide-semiconductor structures (see, e. g., [2-5]). The second direction deals with studies of $\mathrm{Cd}$ and Te emissions during thermal treatment [6-11]. In particular, electronic processes and evaporation kinetics were investigated for $\mathrm{A}^{\mathrm{II}} \mathrm{B}^{\mathrm{VI}}$ ionic crystals in $[6,7]$. It was shown that their chemical composition may change because of different emission intensities of the components. Studies of the constituents evaporation from free and coated $p$-CdTe surfaces showed that the desorption of $\mathrm{Cd}$ and $\mathrm{Te}$ atoms originates at 573 and $873 \mathrm{~K}$, respectively, and the evaporation intensity is essentially reduced when dielectric protective coatings are used [11]. Evaporation from CdTe surface was also investigated by mass-spectroscopic analysis in [10]. A negligibly weak vaporization of $\mathrm{Cd}$ at $T=300$ to $600 \mathrm{~K}$ was explained by outlet of non-stoichiometric cadmium. Higher temperatures caused an abrupt acceleration of $\mathrm{Cd}$ diffusion towards the surface, and the cadmium vaporization was greatly intensified at $T>630 \mathrm{~K}$. Quality of surface treatment also affects the vaporization intensity of the metal atoms from the CdTe surface [9].

At the same time, a little is known about influence of annealing at relatively low temperatures $(T<500 \mathrm{~K})$ on CdTe characteristics. Recently, we showed that photoluminescence (PL) properties of $\mathrm{CdTe}: \mathrm{Cl}$ may be changed essentially if the annealing temperature exceeds $453 \mathrm{~K}$ [12]. This was explained by $\mathrm{V}_{\mathrm{Cd}}$ concentration increase due to the cadmium evaporation and the subsequent formation of the A-centers $\left(\mathrm{V}_{\mathrm{Cd}}-\mathrm{Cl}_{\mathrm{Te}}\right)$. In this paper, we study the low-temperature annealing effect on the optical and electrical properties of semi-insulating $n$-CdTe:Sn.

\section{Experimental}

Tin-doped CdTe single crystals were grown by the modified Bridgman method. Concentration of residual impurities in the elemental cadmium and tellurium was below $10^{-4}$ at. $\%$. Necessary amount of the doping element $\left(C_{\mathrm{Sn}}^{0}=5 \cdot 10^{18} \mathrm{~cm}^{-3}\right)$ was added to the previously synthesized compound. The grown material exhibited $n$-type conductivity with semi-insulating properties. More information on electrical properties of the crystals used in this study can be found in [13].

Electrical measurements (the conductivity $\sigma$ and the Hall coefficient $R_{\mathrm{H}}$ ) were performed on rectangularshaped samples with typical dimensions $12 \times 2 \times 1 \mathrm{~mm}^{3}$. After previous lapping, the samples were grinded, mechanically polished with diamond pastes to obtain a mirror-like surface and then washed in organic solutions 
and deionized water. To form the electric contacts, copper was deposited by electrolytic method, and metallic indium was soldered afterwards. The temperature dependences of $\sigma$ and $R_{\mathrm{H}}$ were measured in the range $T=295$ to $570 \mathrm{~K}$ at rising and lowering the temperature. Each measurement was carried out in 20 min after achieving the determined temperature and then the temperature was varied from 20 to $25 \mathrm{~K}$.

Samples for PL and electrical measurements were taken from the adjacent parts of ingots. PL experiments were performed at $5 \mathrm{~K}$ on cleaved CdTe surfaces using an $\mathrm{Ar}^{+}$laser for excitation. Thereafter the samples were annealed for $20 \mathrm{~min}$ at 453 and $473 \mathrm{~K}$ in evacuated $\left(10^{-3} \mathrm{~mm} \mathrm{Hg}\right)$ quartz ampoules with significantly free volume.

\section{Results and discussion}

Temperature dependences of the Hall coefficient for CdTe:Sn samples cut from the middle (sample C) and end (sample D) parts of an ingot are shown in Fig. 1. The dependences that were taken when rising or lowering the temperature are different. It is seen that sample heating results in a higher conductivity and the difference between $R_{\mathrm{H}}$ values obtained in different cycles of measurements increases proportionally to the upper heating temperature. Annealing the samples occurs at $T \geq 453 \mathrm{~K}$ (vertical dashed lines in Fig. 1) during the electrical measurements. At the lower temperatures, the experimental points can be fit well with straight lines to determine the activation energies $\Delta E$ of the corresponding deep levels. The obtained values of $\Delta E$ given in Fig. 1 for each curve are typical for the semi-insulating CdTe:Sn samples $[13,14]$.

It is known that annealing may change both bulk and surface properties of the crystal. In order to establish which of these processes prevails, the repeat measurements of the $R_{\mathrm{H}}(T)$ dependence were carried out for the same samples but after removing a $130 \mu \mathrm{m}$ surface layer. For both samples the Hall coefficient increases in comparison to the respective values obtained earlier under lowering temperature conditions. It indicates the formation of a low-resistivity near-surface layer at $T \geq 453 \mathrm{~K}$. The comparative $R_{\mathrm{H}}$ measurements for the sample short-term annealed at $573 \mathrm{~K}$ on air and cooled to room temperature showed that the conductivity of this layer is caused by holes. The $\rho(T)$ dependence for the sample with a low-resistivity surface layer is plotted in Fig. 1a (curve 3). The energy position of the active energy level determined by the slope of the $\lg \rho=$ $=f\left(10^{3} / T\right)$ dependence is found to be $\Delta E=0.36 \mathrm{eV}$.

Formation of low-resistivity $p$-type layer in the surface region of the semi-insulating $n$-type material is also confirmed by the time dependences of $\rho$ and $R_{\mathrm{H}}$ measured at $503 \mathrm{~K}$ (Fig. 2). After $4 \mathrm{~h}$ annealing, the resistivity and the Hall coefficient decreased by factors of 1.7 and 4.3 , respectively. Since in the general case the

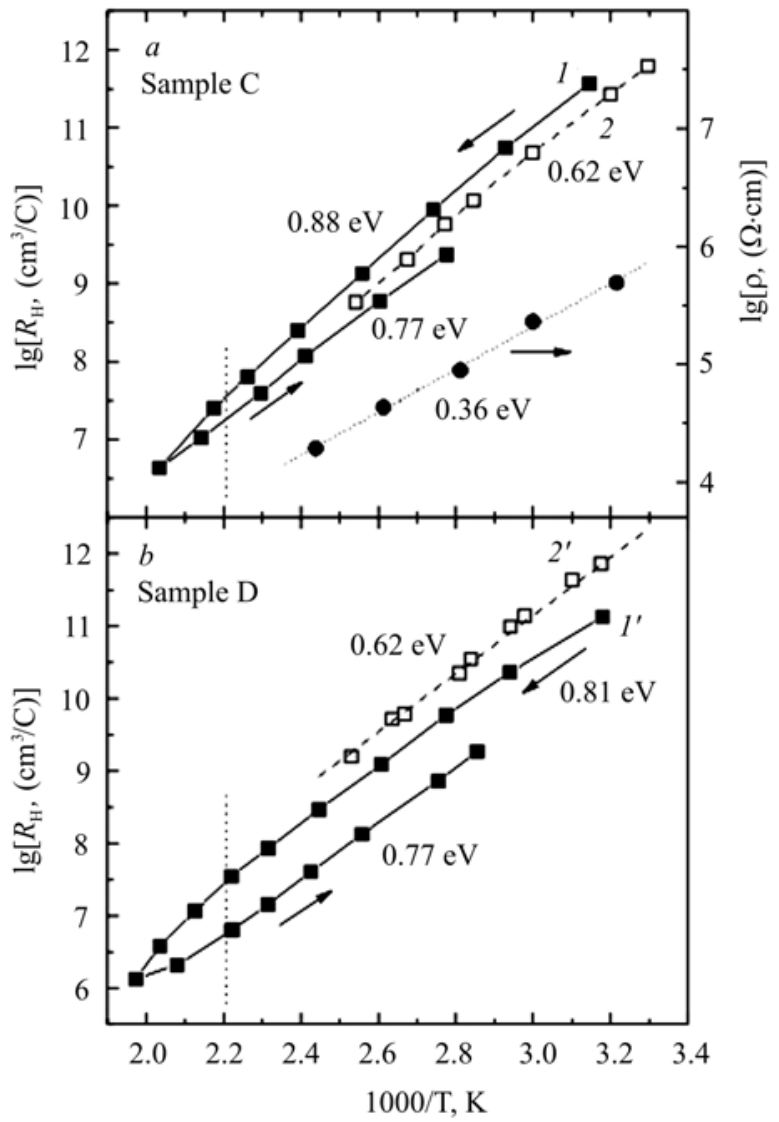

Fig. 1. Temperature dependence of $R_{\mathrm{H}}$ for the CdTe:Sn crystal taken from the middle (a) and end (b) parts of the ingot. Curves 1 and $1^{\prime}$ correspond to the maximal heating temperature of 491 and $503 \mathrm{~K}$, respectively. Arrows indicate the direction of temperature variations. Curves 2 and $2^{\prime}$ are the dependences for the same samples but after removing $130 \mu \mathrm{m}$ surface layer. Curve 3 is the temperature dependence of the resistivity for CdTe:Sn annealed at $573 \mathrm{~K}$.

mixed conductivity is defined as $\sigma=e\left(\mu_{n} n+\mu_{p} p\right)$, the increase in conductivity can be explained by increasing the hole concentration in the near-surface layer. More abrupt decrease of $R_{\mathrm{H}}$ may be related to the fact that the mixed conductivity value depends on the ratio of electron and hole concentrations.

In the case of bipolar conductivity, the Hall coefficient can be written as

$R_{\mathrm{H}}^{\mathrm{B}}=\frac{A}{e n} \cdot \frac{x-b^{2}}{(x+b)^{2}}$,

where $b=\mu_{n} / \mu_{p}$ is the mobility ratio, $A$ is the Hall factor and $x=p / n$ is the ratio of hole and electron concentrations. As for the monopolar (electronic) conductivity, the Hall coefficient is determined as $R_{\mathrm{H}}^{\mathrm{M}}=\frac{A}{e n}$, the ratio $\frac{(x+b)^{2}}{x-b^{2}}$ gives the relative variation of $R_{\mathrm{H}}$ when holes begin to make a contribution to the 


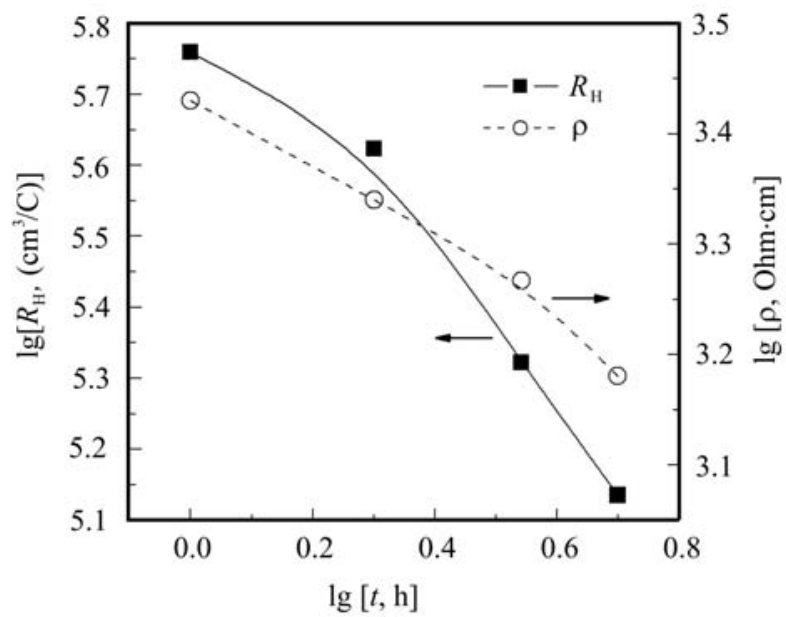

Fig. 2. Time dependences of the Hall coefficient $R_{\mathrm{H}}$ and resistivity $\rho$ for CdTe:Sn (sample D) measured at $T=503 \mathrm{~K}$.

conductivity. The estimations show that, at $b=14$ [15] and $x=1$, the Hall coefficient is decreased by $15 \%$. Taking into account that the measurement accuracy is about $8 \%$, appearance of the hole component can be reliably detected. An essential decrease of the $\sigma R_{\mathrm{H}}$ product observed at lowering the temperature for the samples heated to $T>453 \mathrm{~K}$ also confirms the mixed conductivity in near-surface layers of CdTe.

Formation of the low-resistivity $p$-type layer on the surface of the investigated crystals is caused by the evaporation of $\mathrm{Cd}$ atoms at $T \geq 453 \mathrm{~K}$. As a result, the near-surface layer becomes enriched with cadmium vacancies thus providing the $p$-type conductivity.

As was noted above, on heating CdTe, Cd desorption has been observed by many authors [6-11]. However, the temperature at which the vaporization begins has not been reliably defined yet. It is due to both different in sensitivity of the applied experimental methods and properties of the crystals under investigations (surface treatment, carrier concentration, etc.). Our results are in good agreement with results of the work [12], where the enhancement of exciton luminescence related to complex defects with $\mathrm{V}_{\mathrm{Cd}}$ participation was found after the vacuum annealing of $\mathrm{CdTe}: \mathrm{Cl}$ at $T \geq 453 \mathrm{~K}$.

Fig. 3 shows the low-temperature (5 K) PL spectra of CdTe:Sn samples before and after the short-time (20 min) vacuum annealing at 453 and $473 \mathrm{~K}$. Three lines can be resolved in the exciton region of the spectra: the lines $\left(\mathrm{A}^{\circ}, \mathrm{X}\right)$ at around $1.590 \mathrm{eV}$ as well as ones $\left(\mathrm{D}^{\circ}\right.$, $\mathrm{X})$ at $1.593 \mathrm{eV}$ are typical for CdTe crystals and assigned to the excitons bound to neutral acceptors and donors, respectively. A feature close to $1.591 \mathrm{eV}$ is observed at the high-energy side of the $\left(\mathrm{A}^{\circ}, \mathrm{X}\right)$ line. For chlorine-doped $\mathrm{CdTe}, 1.591 \mathrm{eV}$ line is assigned to the excitons bound to the acceptors $\left(\mathrm{V}_{\mathrm{Cd}}-2 \mathrm{Cl}_{\mathrm{Te}}\right)$ [16]. To our knowledge, the origin of this line in CdTe doped with IV group elements is yet to be established. However, we may assume that, similarly to $\mathrm{CdTe}: \mathrm{Cl}$, the acceptor that is responsible for this line is also formed by $\mathrm{V}_{\mathrm{Cd}}$ and two shallow donors. This assumption is supported by the fact that the relative intensity of $1.591 \mathrm{eV}$ line increases for samples cut from the last-to-freeze part of the ingot in which accumulated are the background donor impurities with the segregation coefficient less than unity ( $\mathrm{In}, \mathrm{Cl}$, $\mathrm{Al}$, etc.).

Similarly to undoped CdTe, intensity of the edge PL $(1.50-1.56 \mathrm{eV})$ is much weaker in comparison to that of the $\left(\mathrm{A}^{\circ}, \mathrm{X}\right)$ line. The emission close to $1.4 \mathrm{eV}$ connected with radiation of the electrons localized on shallow donors to A-centers is not detected because of the low concentration of the D-A pairs.

The thermal treatment at $T=453 \mathrm{~K}$ does not influence neither shape nor the intensity of the exciton lines but results in some decrease of the edge PL intensity. Annealing at the higher temperature $(473 \mathrm{~K})$ leads to more distinct reduction of the edge emission. At the same time, a redistribution of the relative intensities of the different exciton lines is observed. In particular, the intensity of $1.591 \mathrm{eV}$ line is greatly enhanced with respect to the $\left(\mathrm{A}^{\circ}, \mathrm{X}\right)$ line. Since it depends on the concentration of the $\mathrm{V}_{\mathrm{Cd}^{-}}$-related complexes, as

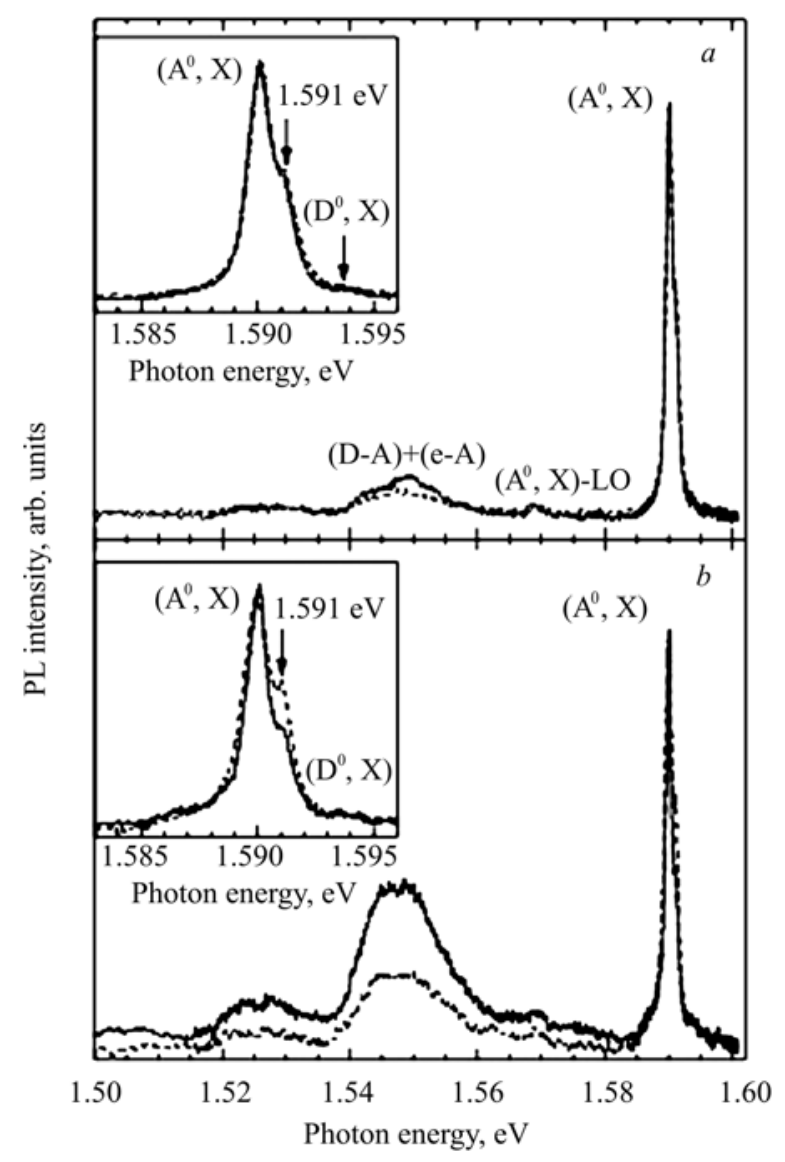

Fig. 3. PL spectra $(T=5 \mathrm{~K})$ of CdTe:Sn before (solid lines) and after (dashed lines) the vacuum annealing for $20 \mathrm{~min}$ at 453 (a) and $473 \mathrm{~K}$ (b). Insets with the enlarged scale show the respective exciton regions of the spectra. 
mentioned above, we may conclude that the annealing at $473 \mathrm{~K}$ results in an increase of the $\mathrm{V}_{\mathrm{Cd}}$ concentration in the near-surface layer of CdTe:Sn due to the cadmium evaporation. An additional evidence of this conclusion comes from low-energy broadening the $\left(\mathrm{A}^{\circ}, \mathrm{X}\right)$ line in the annealed samples (see inset in Fig. 3b). Recent results by Song et al. showed that the $\left(\mathrm{A}^{\circ}, \mathrm{X}\right)$ line is actually formed by the excitons bound to different acceptors: $\mathrm{Cu}_{\mathrm{Cd}}(\mathrm{PL}$ line maximum at $1.5896 \mathrm{eV}), \mathrm{Ag}_{\mathrm{Cd}}$ $(1.5885 \mathrm{eV})$ and $\mathrm{V}_{\mathrm{Cd}}(1.5892 \mathrm{eV})$ [17]. Therefore the low-energy broadening observed in our experiments reflects increasing the concentration of cadmium vacancies.

\section{Conclusions}

We studied the influence of the low-temperature thermal treatment at $T \leq 573 \mathrm{~K}$ on the electrical and luminescent properties of the $n$-type semi-insulating CdTe:Sn crystals. It was ascertained that emission of $\mathrm{Cd}$ atoms from surface originates at $T \geq 453 \mathrm{~K}$, which results in the increase of the $\mathrm{V}_{\mathrm{Cd}}$ concentration in the near-surface layer and thus enhances the hole contribution to the conductivity. Increasing both the annealing duration and temperature leads to formation of the near-surface layer with $p$-type conductivity. Some carrier concentration changes in the bulk of the crystals observed for our thermally treated samples may be explained by diffusion flow of the metal atoms towards the surface because of $\mathrm{Cd}$ desorption from the crystal surface. It affects an equilibrium in the system of intrinsic and impurity defects and therefore the compensation conditions.

\section{References}

1. D.V. Korbutyak, S.V. Melnychuk, E.V. Korbut, and M.M. Borysyuk, Cadmium telluride: defect-impurity states and detector properties, Ivan Fedorov, Kyiv (2000).

2. T.L. Chu and Si.S. Chu, Surface passivation and oxidation of cadmium telluride and properties of metal-oxide-CdTe structures // J. Appl. Phys. 58 (8), p. 3206-3210 (1985).

3. M. Suita and T. Taguchi, Thermal oxidation of CdTe surface and the properties of MDS-diodes // Nucl. Instrum. Meth. A 283, p. 268-273 (1989).

4. G.A. Il'chuk, V.I. Ivanov-Omskii, V.Yu. Rud', Yu.V. Rud', R.N. Bekimbetov, and N.A. Ukrainetz, Preparation and photoelectrical properties of oxide CdTe structures // Fiz. Tekhn. Poluprov. 34(9), p. 1099-1102 (2000).
5. J. Garcia-Garcia, J. Gonzales-Hernandes, J.G. Mendoza-Alvarez, Photoluminescence characterization of the surface layer of chemically etched CdTe // J. Appl. Phys. 67(8), p. 3810-3814 (1990).

6. G.Ya. Pikus and G.Ye. Chaika, Role of electronic processes in vaporization mechanism and in formation of the composition of binary semiconductor compound with ionic bonding // Ukr. Fiz. Zhurn. 18(6), p. 931-939 (1973).

7. G.N. Tal'nova and G.Ya. Pikus, Electronic processes and kinetics of vaporization of ionic crystals in vacuum // Zhurn. Fiz. Khim. L11(12), p. 3107-3112 (1978).

8. T.B. Fedorova, A.V. Vishnyakov, and P.V. Kovtunenko, Composition of the products and kinetics of low-temperature oxidation of CdTe // Izv. AN SSSR: Neorg. Mater. 23(6), p. 912-916 (1987).

9. V.B. Matulskii, B.V. Pavlyk, A.V. Savitskii, and N.A. Tzal', Investigation of gas emission at annealing of irradiated cadmium telluride single crystals // Fiz. Elektronika 35, p. 63-67 (1987).

10. V.B. Matulskii, B.V. Pavlyk, A.V. Savitskii, and V.R. Burachek, Influence of dislocation structure on emission of $\mathrm{Cd}$ from CdTe // Elektr. Tekhn.: Materialy 239(2), p. 71-72 (1989).

11. T. Yoshikawa, K. Yokota, S. Tamura, S. Katayama, I. Kimura, S. Ishihara, Decomposition of bare and covered cadmium telluride during annealing // Ann. Rep. Res. Reactor Inst. Kyoto Univ. 15, p.123-128 (1982).

12. N.D. Vakhnyak, S.G. Krylyuk, S.Yu. Paranchych, and M.D. Raranskii, Influence of active external treatments on impurity-defect states in CdTe:Cl // Bulletin of Chernivtsi National University: Physics and Electronics 133, p. 86-94 (2002).

13. O.A. Parfenyuk, A.V. Savitskii, P.A. Pavlin, and A.L. Al'bota, Compensating effect of Sn dopant in cadmium telluride // Izv. AN SSSR: Fizika N 4, p. 6669 (1986).

14. J. Franc, M. Fiederle, V. Babentsov, A. Fauler, K.W. Benz, and R. James, Defect structure of Sndoped CdTe // J. Electron. Mater. 32(7) p. 772-778 (2003).

15. Physics and Chemistry of II - VI Compounds, ed. by S.A. Medvedev, Mir, Moscow (1970).

16. H.-Y. Shin and C.-Y. Sun, Photoluminescence spectra of Cl-doped CdTe crystals // J. Crystal Growth 186, p. 354-361 (1998).

17. S.H. Song, J.F. Wang, and M. Isshiki, Cd-vacancyrelated excitonic emission in CdTe // Ibid. 257, p. 231-236 (2003). 